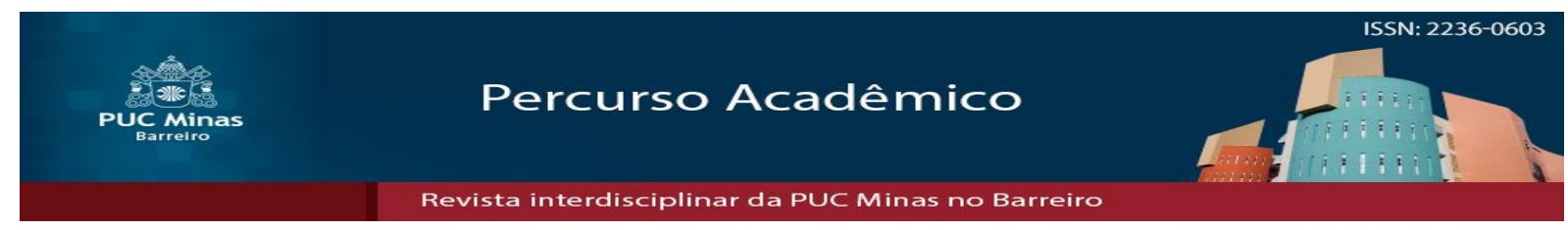

\title{
O impacto da Global Financial Crisis (GFC) sobre os Recursos Humanos em empresas multinacionais na Irlanda
}

\section{The Global Financial Crisis impact on the Human Resource Management in multinationals in Ireland}

\author{
Charles Alves Castro ${ }^{1}$ \\ Felipe Gouvêa Pena ${ }^{2}$
}

\section{Resumo}

O presente artigo tem por objetivo analisar e revisar o impacto da crise financeira global sobre a gestão de recursos humanos nas empresas multinacionais na Irlanda. Foi realizada uma revisão da literatura, tomando-se como referência o estudo de Gunningle, Lavelle e Monaghan (2013) e os dados coletados em jornais, revistas, livros e periódicos científicos. A grande contribuição do artigo está relacionada ao fato de trazer um caso, a Irlanda, para demonstrar que em momentos de crises, os gestores da área de RH podem e devem ser vistos como key-players dentro das empresas, atuando de forma estratégica. Torna-se necessário que os gestores de RH, para se comunicar de forma eficaz, construam boas relações e estejam atentos a difícil tarefa de equilibrar os anseios individuais e organizacionais. Tratar o caso específico da Irlanda traz limitações do ponto de vista das generalizações, mas funciona como uma ilustração de alternativas gerenciais às empresas impactadas por "turbulências" econômicas, seja para validação das ideias propostas ou para a construção de novas.

Palavras-chave: Crise econômica. Recursos humanos. Multinacionais. Irlanda.

\section{Abstract}

This paper aims to analyze and review the global financial crisis impact on human resource management in multinational corporations in Ireland. A literature review was done as the main catalyst for this research, in addition the study of Gunningle, Lavelle and Monaghan (2013) and the secondary data collected in newspapers, magazines, books and scientific journals. The key contribution of this article is to highlight Ireland as a case of study in order to demonstrate that in times of crisis HR managers can and

\footnotetext{
Artigo recebido em 24 de Março de 2019 e aprovado em 07/06/2019

${ }^{1}$ Mestrando em Marketing pela Universidade de Bari Aldo Moro na Itália . Mestre em Gestão de RH pela Universidade de Limerick na Irlanda. Graduado em Administração de Empresas e Processos Gerenciais pela Universidade Newton Paiva, Brasil. E-mail: charlesturcastro@yahoo.com.br

${ }^{2}$ Mestre em Administração pela Universidade Federal de Minas Gerais (UFMG), Especialista em Gestão de Pessoas e Especialista em Metodologias Ativas para a Educação pela Pontifícia Universidade Católica de Minas Gerais (PUC MINAS), Graduado em Administração pelo Centro Universitário Newton Paiva, Brasil. E-mail: felipegouveap@hotmail.com
} 
should be seen as key players within companies, acting in a strategic role. It is necessary for HR managers to communicate effectively, build good relationships and pay attention to a variety of difficult tasks in order to balance an individual and organizational desires. Addressing the specific case of Ireland there are some limitations from a general point of view, despite it works as an illustration of managerial alternatives to companies impacted by economic "turbulences", whether to validate the proposed ideas cited and to develop new ones.

Key-words: Economic crisis. Human resources. Multinationals. Ireland.

\section{Contextualização}

A crise financeira desencadeada em 2007 nos Estados Unidos impactou todas as economias do mundo, sendo assim conhecida como "'Global Financial Crisis (GFC)". Causada por uma série de problemas no sistema financeiro americano foi a pior existente desde a recessão de 1930, afetando diretamente os países desenvolvidos e em seguida expandiu-se por todo planeta. Desta forma, teve um impacto significante nos investimentos e negócios de modo geral resultando em um mundo cada vez mais interconectado (OECD 2009a). A Europa, por exemplo, foi fortemente afetada, destacando países como Portugal, Irlanda, Itália, Grécia e Espanha (PIIGS) sendo os mais impactados, sofrendo uma intensa crise com elevados índices de desemprego e cortes em todos os setores públicos e privados, além de ter instigado inúmeras empresas a falência mutua.

O principal objetivo deste artigo é analisar e revisar o impacto da crise financeira global sobre a gestão de recursos humanos nas empresas multinacionais na Irlanda. O primeiro ponto a ser discutido é a crise financeira na Irlanda e o seu atual status no ponto de vista do departamento de RH instalado em multinacionais; Em seguida o tema discorre sobre as peculiaridades da gestão de recursos humanos como: treinamento e desenvolvimento (T\&D), o papel do gestor, as funções gerais do $\mathrm{RH}$, salários, cadeia de benefícios e relações sindicais. Por fim, foi analisado o impacto da crise financeira global sobre a gestão de RH em multinacionais na Irlanda.

Para evidenciar o objetivo geral deste artigo foi realizada uma pesquisa qualitativa a partir de diversos textos publicados em jornais, livros, revistas e periódicos científicos, para que fosse possível traçar, com maior clareza, as perspectivas gerenciais em relação às práticas de recursos humanos em momentos de crise. Além disso, foi examinada uma importante pesquisa conduzida por três pesquisadores irlandeses 
(Gunningle, Lavelle e Monaghan) realizada em 2013 sobre o impacto da crise financeira global na gestão de RH em multinacionais na Irlanda. Portanto, toma-se esse estudo como eixo norteado do presente artigo. Nesses termos, a intenção deste artigo é discutir o desenvolvimento e as mudanças das práticas em gestão de RH durante a crise financeira global, principalmente no contexto da economia Irlandesa, considerando que a recessão afetou diretamente o setor de RH, positivamente e negativamente. Além disso, o material que será apresentado discorre sobre "saídas estratégicas" e "alternativas criativas" que as empresas estudadas lançaram mão para amenizar o impacto de tal crise econômica.

\section{Irlanda, Crise Financeira Global e Multinacionais}

O cenário macroeconômico Irlandês pode ser considerado sólido, possuidor de um Produto Interno Bruto (PIB) de US\$ 2.077 trilhões em 2013, tendo uma crescente de 2.5\% em 2014 e 3.3\% em 2015 (IDA, 2015; WORLD BANK 2015). Além disso, o banco central irlandês (THE IRISH CENTRAL BANK, 2016) elevou suas previsões econômicas para a Irlanda e acreditava que o PIB iria crescer 4,1\% em 2016.

Todavia, a crise econômica mundial atingiu a Irlanda fortemente a partir de 2007 (DELLEPIANE; HARDIMAN, 2011), em meio à crise financeira global que afetou todas as economias ao redor do mundo. Na Irlanda constatou-se a queda do PIB nominal acumulada de 21\% a partir de Q4 2007 para Q3 2010. Essa situação classificou a Irlanda como um dos países mais afetados em termos de desempenho de resultados econômicos durante esse período (LANE; MILESI-FERRETTI 2010). Mesmo com as adversidades, alguns posicionamentos ajudaram o país a passar pelas "tormentas".

É notório que a Irlanda tem sido um país de grande importância para os investimentos diretos estrangeiros (IDE) mesmo anterior à crise, possuidor de uma grande escala de atividade produtiva compartilhada e de exportações, tanto na manufatura quanto em serviços gerados pelas multinacionais estrangeiras localizadas na ilha. Durante a recessão foi necessário manter elevados níveis de investimentos para manter a Irlanda um país atraente para os investimentos diretos estrangeiros (IDE), evitando assim uma grande queda nas exportações (GODART et al, 2011). 
Assim, os principais aspectos para a atração de investidores estrangeiros para a ilha incluem um baixo nível de impostos sobre os lucros das empresas, financiamentos/subsídios e outras formas de incentivos financeiros, bem como, uma maior preocupação com a qualidade de vida no trabalho e suporte aos funcionários (GUNNIGLE; HERATY; MORLEY, 2011). Aspectos demográficos, educação, desenvolvimento de habilidades e competências, salários justos, custo de vida, baixa dos impostos, facilidade em adquirir propriedades e infra-estrutura também são razões que influenciam os investimentos na Irlanda (IDA, 2015).

A evidência do excelente desempenho das estratégias e alternativas criativas do governo irlandês para atrair capital estrangeiro foi comprovada em 2013, pois o total de exportações da ilha foi avaliado em $€ 185$ bilhões, $€ 2$ bilhões a mais, comparado com o ano de 2012 que fechou em $€ 183$ bilhões. Os principais mercados consumidores são da Europa e os Estados Unidos, sendo os principais setores exportadores: farmacêutico e produtos químicos, serviços de tecnologia da informação, serviços empresariais, serviços financeiros e seguros, alimentação e dispositivos para a área da saúde (IDA, 2015).

A Irlanda abriga atualmente "sete das 10 maiores empresas globais de tecnologias de informação e comunicação, 15 das 25 maiores empresas globais de tecnologia médica, nove das 10 principais companhias farmacêuticas globais, mais de 250 instituições financeiras globais", além de um setor de jogos e mídias sociais em avanço (GUNNIGLE; LAVELLE; MONAGHAN 2013, p. 215). Por isso, torna-se oportuno atrair as multinacionais, a fim de gerar empregos, tornando a sua economia produtiva e criando um ambiente multicultural. A Irlanda tem a $5^{\mathrm{a}}$ maior proporção do estoque de investimentos diretos estrangeiros (IDE) em relação ao PIB na OCDE (Organização de Cooperação e de Desenvolvimento Econômico) e a maior proporção de emprego nas filiais estrangeiras nos setores da indústria e serviços (OCDE, 2010) o que fortalece o país como um território de oportunidades.

\section{Recursos Humanos e a GFC}

Durante a recessão, o declínio da economia impactou diretamente as funções voltadas para o RH (Recursos Humanos). Algumas tendências foram examinadas e 
observadas pelos pesquisadores do tema, concluindo que as empresas enfrentaram e consequentemente adotaram um conjunto de medidas, como: a proibição de horas extras, congelamento sobre o processo de recrutamento e seleção (R\&S), congelamento no processo de treinamento e desenvolvimento (T\&D), demissões generalizadas, perdas de emprego, congelamento da cadeia de benefícios e salarial e cortes salariais (CAMERON et al, 1991).

Todas as alternativas citadas anteriormente também foram adotadas durante outras recessões, como, por exemplo, na recessão de 1980 na Inglaterra. Alguns estudiosos avaliaram amplamente um conjunto de evidências que fogem das principais alternativas usadas pelas empresas, eles analisaram o impacto da crise econômica no Reino Unido (UK) no início de 1980, enfatizando o poder, o papel e a estrutura dos departamentos de pessoal nas empresas. O foco desta investigação discorreu sobre as seguintes hipóteses: a) se aumentou o status da função de RH, abrindo novas oportunidades para que possa levar a mudança organizacional e reestruturação do departamento; b) se reduziu a necessidade de específicos aspectos do $\mathrm{RH}$, relacionado à suas principais atividades, por exemplo, recrutamento e seleção que seria menos importante para as empresas; c) se causou a marginalização da gestão de RH, abrindo oportunidades para tornar a gestão do departamento mais machista; ou d) não levou a nenhuma mudança significativa para o papel ou os valores da função de RH. Legge (1987) concluiu que, embora a incidência pudesse ser encontrada do departamento de RH estar perdendo o poder dentro das organizações e a gestão se tornando cada vez mais machista, as tendências dominantes pareciam estar permanecendo mais ou menos o mesmo sendo um resultado da recessão (ROCHE; TEAGUE 2012).

Por outro lado, Gunnigle, Lavelle e Monaghan (2013) analisou o impacto da crise financeira asiática de 1997 sobre o setor de $\mathrm{RH}$ fornecendo elementos razoavelmente recentes a este respeito.Assim constatando que as principais alternativas usadas pelas empresas foram: congelamentos e cortes salariais, redução de bonificações, alocações de remuneração variável, congelamento do processo de recrutamento e seleção, uso de trabalho atípico, alterações no sistema de gestão de desempenho e congelamento do processo de treinamento e desenvolvimento (BENSON, 1998; KAMOCHE， 2003; SMITH; ABDULLAH， 2004; FODORANDPOOR， 2009; GENNARD, 2009 apud GUNNIGLE; LAVELLE; MONAGHAN, 2013) 
Portanto, há muitos exemplos sobre o impacto da crise financeira global e outras recessões sobre os recursos humanos e suas respectivas atividades. Gunnigle, Lavelle e Monaghan (2013) examinou em sua pesquisa " Resistindo à tempestade? Multinacionais e a Gestão de Recursos Humanos atravessando a Crise Financeira Global " a vigência de quatro proposições econômicas típicas sobre o impacto esperado da recessão sobre a prática da gestão de RH em multinacionais na Irlanda, tais como: pessoal, remuneração e benefícios, relações industriais/sindicais e o papel da função de RH. Tais proposições (GUNNIGLE; LAVELLE; MONAGHAN, 2013, p.216) sobre o impacto esperado da crise financeira global sobre a prática da gestão de RH em multinacionais na Irlanda, são:

- P1. Pessoal: haverá um declínio dramático no nível de recrutamento e seleção, desta forma as organizações terão de ajustar os níveis de pessoal reduzindo sua demanda, ao mesmo tempo haverá uma exploração de "padrões de pessoal" mais flexíveis e mais baratas, por exemplo: de curto tempo de trabalho, trabalho temporário, licença sem vencimento e demissões. Além disso, a escala das atividades de T\&D irão diminuir consequentemente diminuindo custos.

- P2. Remuneração e benefícios: os níveis de remuneração e benefícios tendem a cair, pois as empresas procuram obter reduções de custos de trabalho.

- P3. Relações Industriais/Sindicais: o gestor irá explorar o poder de negociação, focando nas fraquezas dos sindicatos e dos trabalhadores, prosseguindo por uma série de mudanças em termos e condições de práticas trabalhistas, seja através de negociação de concessões ou tomada de decisões unilaterais.

- P4. Papel da função de RH: o papel, a influência e as considerações da função de RH irão diminuir em importância estratégica pois as preocupações financeiras e operacionais passaram a dominar a agenda gerencial. Esta preposição já é clara desde muitos anos sobre a credibilidade da contribuição de RH para os critérios de sucesso organizacionais convencionais (Legge, 2005) - tomadas de decisões são mais evidentes durante a recessão econômica.

Gunnigle, Lavelle e Monaghan (2013) encontraram em sua pesquisa uma magnitude de provas que indicam que as multinacionais têm sido o precursor das 
organizações envolvidas em programas de reestruturação multidimensionais em resposta à crise financeira global, incorporando muitas iniciativas no domínio da gestão de recursos humanos. Em termos de pessoal, eles descobriram que uma variedade de ações tem sido realizada dentro de multinacionais em resposta a GFC. De acordo com o ECM (Enterprise Content Management) cerca de 14.546 postos de trabalho foram criados, enquanto 27.317 empregos foram perdidos, eles também encontraram evidências sobre a extensão do trabalho há tempo parcial e temporário em empresas multinacionais.

Figura 1 - Types of Restructuring and Job Losses and Gains in MNCs in Ireland

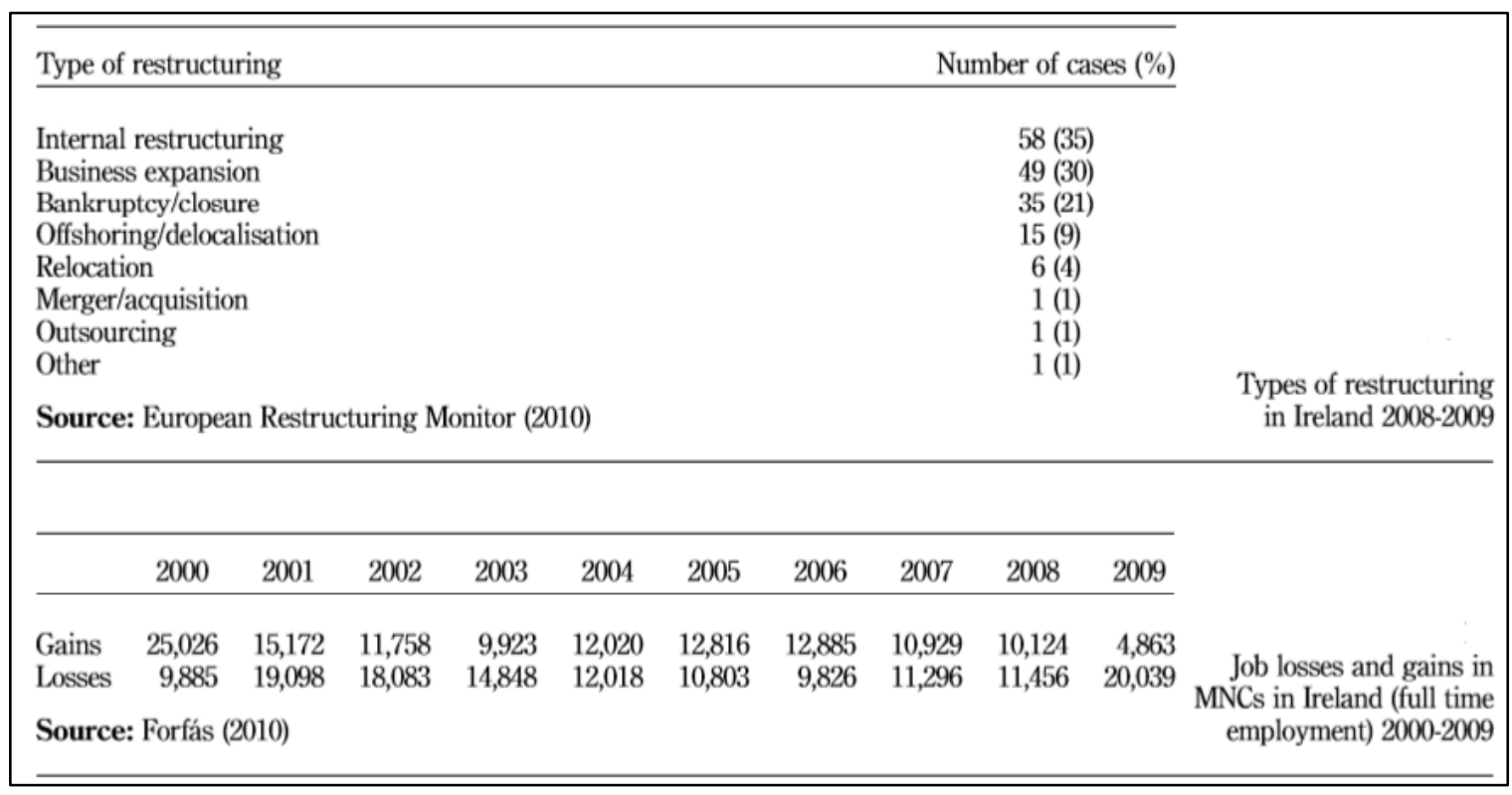

Fonte: Gunnigle, Lavelle e Monaghan (2013)

Ainda, um aspecto importante é em relação Treinamento e Desenvolvimento ( $T$ \& D) que confirma que as despesas do mesmo foram submetidas a cortes significativamente, portanto, a atividade e as despesas de T\&D é cada vez mais sistemática, pois os custos são extremamente reduzidos (CIPD, 2010; HAYGROUP, 2009 apud GUNNIGLE; LAVELLE; MONAGHAN. 2013). Outro ponto é que o treinamento foi mais afetado do que outras atividades devido à queda na atividade de $\mathrm{R} \& \mathrm{~S}$.

Em relação à cadeia de remuneração e benefícios, as empresas fizeram mudanças nos seus sistemas de benefícios, principalmente relacionados aos planos de pensão e os regimes de participação nos lucros. As multinacionais foram o setor de 
empresas que menos sofreu aumentos salariais durante a crise econômica na Irlanda, comparado com outros tipos de organizações. Em termos de relações industriais/sindicais a prerrogativa de gestão aumentou substancialmente. Esta mudança no equilíbrio do poder está sendo usada em empresas multinacionais, sendo empurradas através de iniciativas de reestruturação e mudanças em termos e condições de empregabilidade. Além disso, o interesse dos funcionários de se juntar aos sindicatos aumentou, consequentemente, oferecem a perspectiva para o movimento operário de inverter o declínio no número de membros. Portanto, os sindicatos possuem limitada margem de manobra na prevenção de cortes de empregos.

De acordo com os pesquisadores, referindo-se as funções de $\mathrm{RH}$, eles não encontraram qualquer evidência generalizada para sugerir que os setores financeiros e de custos estão no centro das atenções. A função de RH desempenhou um papel fundamental na concretização dos resultados durante a crise econômica dentro das multinacionais. De acordo com as multinacionais as tomadas de decisões estratégicas relacionadas a temas, como: reestruturação, downsizing e alterações na remuneração e benefícios eram geralmente geridos através da função de RH (GUNNIGLE; LAVELLE; MONAGHAN. 2013).

Além disso, os autores defendem que a função de RH foi afetada pela crise financeira, tanto positiva quanto negativamente. Pois, os gestores de $\mathrm{RH}$, por exemplo, demonstraram a sua capacidade para tomar decisões estratégicas e de resolver problemas. Estudos descobriram que os gerentes de RH ganharam influência e destaque nos processos de tomada de decisão em muitas organizações durante a crise, assim, o departamento de RH dentro das empresas aumentou seu nível hierárquico, mudando do nível tático para o estratégico.

Em termos de empresas multinacionais na Irlanda o mais importante legado para eles tem sido a difusão de novas técnicas de RH, por exemplo, em áreas como testes de seleção, métodos de treinamento, sistema de recompensa, táticas de negociações e de comunicações (GUNNIGLE; LAVELLE; MONAGHAN, 2013). Além disso, durante a crise o papel do departamento e do gestor de RH foi destacado. O primeiro, como um setor essencial para as empresas, principalmente para as multinacionais, e o segundo, os verdadeiros agentes de mudanças e possível "polinizadores" de boas práticas. 


\section{Considerações finais}

Após a exposição dos dados e das implicações demonstradas pela literatura, conclui-se que em tempos difíceis relacionados à economia, é essencial que os custos das empresas sejam reduzidos (as vezes a quase zero), assim difíceis decisões têm de ser feitas, por exemplo em relação às atividades de treinamento e desenvolvimento que geralmente são mais afetadas. Os gerentes de RH devem negociar em meio estas situações criando alternativas estratégicas e criativas que permitam o crescimento de seus talentos, a fim de garantir a estabilidade da empresa e dos postos de trabalho.

Outro ponto a ser notado é que as iniciativas tomadas pelas multinacionais na Irlanda durante a crise financeira global, interligado aos organismos de governo irlandês, podem ser um exemplo para os países que enfrentam ou enfrentarão crises. Além de ser um estudo adequado para todas as empresas possibilitando o desenvolvimento e correção de lacunas a serem preenchidas e discutidas dentre as funções de RH.

Conclui-se que os gerentes de RH podem e devem ser vistos como key-players dentro das empresas afetadas durante momentos de turbulência, como foram durante a GFC, atuando de forma estratégica. Torna-se necessário que os gestores de RH, para se comunicar de forma eficaz, construam boas relações e estejam atentos a difícil tarefa de equilibrar os anseios individuais e organizacionais. Por fim, reafirma-se que a gestão coerente de recursos humanos é um catalisador de sobrevivência das empresas, capaz de auxiliar no enfrentamento dos desafios atuais, de curto e longo prazo diante de crises econômica financeiras.

Espera-se que esse trabalho suscite reflexões sobre o papel da área de recursos humanos em momentos de instabilidade e mudanças organizacionais, pois não é mais viável considerar o RH como um mero braço técnico da gestão empresarial. O texto aqui apresentado trata-se da ilustração de um contexto específico, mas serve de provocação a novos questionamentos sobre o posicionamento, os processos e as práticas emanadas das organizações em relação às pessoas, seja no âmbito público ou no privado. 


\section{Referências}

Dellepiane, S; Hardiman, N. (2011) 'Governing The Irish Economy: A triple Crisis', University College Dublin, Geary Institute [online], available: http://researchrepository.ucd.ie/bitstream/handle/10197/4931/Dellepiane_and_Hardima $\underline{\text { n\%2c_Governing the_Irish_Economy_-_A_Triple_Crisis.pdf? sequence }=1}$

IDA Ireland (n. d.) [online] available http://www.idaireland.com/

Iwulska, A. (2012) With contributions from Quillin, B, For the World Bank report "Golden Growth: Restoring the lustre of the European economic model" by Gill and Raiser (2012) Published by Wold Bank [online] available: http://siteresources.worldbank.org/ECAEXT/Resources/2585981284061150155/7383639-1323888814015/8319788-1324485944855/benchmarks.pdf Lane, P. (2011) The Irish Crisis available [online] http://www.philiplane.org/lanecrisis2010.pdfPublishedby Trinity College Dublin and CEPR

Godart, O.; Gorg, H., Hanley, A. (2011) Surviving the Crisis: Foreign Multinationals vs Domestic Firms in Ireland available [online] Published by IZA

Gunnigle, P., Heraty, N. and Morley, M. (2011) Human Resource Management in Ireland, $4^{\text {th }}$ ed., Dublin: Gill \&Macmillan

Gunnigle, P., Heraty, N. and Morley, M. (2002) Human Resource Management in Ireland, $2^{\text {th }}$ ed., Dublin: Gill \&Macmillan

Gunnigle, P., Lavelle, J., Monaghan, S. (2013) ''Weathering the storm? Multinational Companies and Human Resource Management through the Global Financial Crisis", International Journal of Manpower, vol. 34, pp. 214-231

Gunter, S. Bjorkman, I. (2006) Handbook of Research in International Human Resource Management, UK: Edward Elgar Publishing Limited

OECD (2015) [online], available: http://www.oecdbetterlifeindex.org/topics/jobs/ Roche, W., Teague, P. (2012) ''Business Partners and Working the Pumps: Human Resource Managers in the Recession', Human Relations, 65(10), 1333-1358. 
Rowley, C., Warner, M., 2004. The Asian financial crisis: The impact on human resource management. International studies of management and organization, 34 (1), 39.

Teague. P., Roche, W. (2013) ''Recessionary Bundles: HR Practices in the Irish Economic Crisis', Human Resource Management Journal, p. 17

Tower Watson (2014) A new strategic HR Model: Preparing for What's Next [online], available: file:///C:/Users/Charles\%20Castro/Desktop/a-new-strategic-hr-model-towerswatson.pdf

Tower Watson (n. d.) [online], available: http://www.towerswatson.com/enIE/Insights/IC-Types/Survey-Research-Results/2014/06/2014-hr-service-delivery-andtechnology-survey 